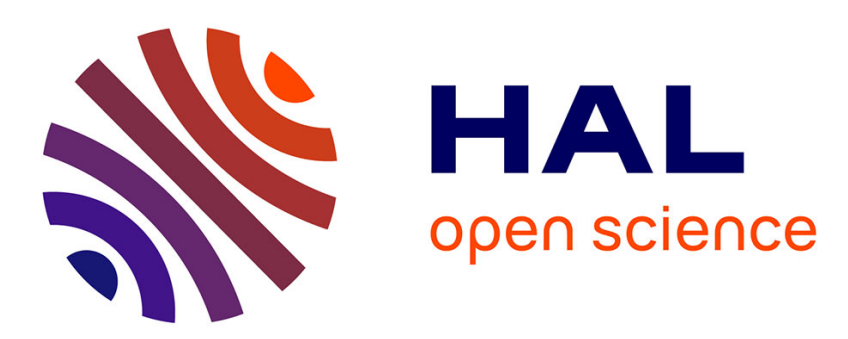

\title{
Remarques à propos d'une analogie entre dislocations de translation dans les smectiques et lignes de courant
}

\author{
M. Kléman
}

\section{To cite this version:}

M. Kléman. Remarques à propos d'une analogie entre dislocations de translation dans les smectiques et lignes de courant. Journal de Physique Lettres, 1976, 37 (4), pp.93-96. 10.1051/jphyslet:0197600370409300 . jpa-00231245

HAL Id: jpa-00231245

https://hal.science/jpa-00231245

Submitted on 1 Jan 1976

HAL is a multi-disciplinary open access archive for the deposit and dissemination of scientific research documents, whether they are published or not. The documents may come from teaching and research institutions in France or abroad, or from public or private research centers.
L'archive ouverte pluridisciplinaire HAL, est destinée au dépôt et à la diffusion de documents scientifiques de niveau recherche, publiés ou non, émanant des établissements d'enseignement et de recherche français ou étrangers, des laboratoires publics ou privés. 


\title{
REMARQUES A PROPOS D'UNE ANALOGIE ENTRE DISLOCATIONS DE TRANSLATION DANS LES SMECTIQUES ET LIGNES DE COURANT
}

\author{
M. KLÉMAN \\ Laboratoire de Physique des Solides, Bâtiment 510, Université Paris-Sud, 91405 Orsay, France
}

(Reçu le 5 février 1976, accepté le 13 février 1976)

\begin{abstract}
Résumé. - On étend au cas des smectiques déformés selon de grandes courbures (élasticité covariante non linéaire) l'analogie entre lignes de courant et dislocations de translation proposée par Pershan. On obtient une expression de la force de Peach et Koehler s'exerçant sur une ligne et on met l'énergie d'une ligne et l'énergie d'interaction entre lignes sous la forme d'intégrales de surface. On discute de la validité de ces expressions en élasticité non linéaire.
\end{abstract}

\begin{abstract}
The analogy proposed by Pershan between dislocation lines and electric current lines is extended to the case of strongly curved layers (non-linear covariant elasticity). A simple expression is obtained for the Peach and Koehler configurational force; the self-energy of a line and the energy of interaction between lines are given as surface integrals. The validity of theses expressions is discussed with respect to the non-linear character of the theory.
\end{abstract}

L'élasticité des smectiques est bien établie dans le cas où l'on ne s'intéresse qu'aux petites déformations au voisinage de la structure en couche planaire [1], et en particulier on connaît bien, dans cette approximation, les champs de distorsions et de contraintes dus à une boucle de dislocation [2] : le caractère linéaire de la théorie permet d'ailleurs d'introduire sans difficultés des concepts bien connus dans le cas des dislocations dans les solides, comme les notions de contraintes internes, de forces de configuration (force de Peach et Koelher), d'énergie de ligne, d'énergie d'interaction entre lignes, etc... et ceci de manière très analogue au cas des solides [2].

Plutôt que de chercher à comparer dislocations dans les solides et dans les smectiques, on peut établir la théorie linéaire des dislocations dans les smectiques en se fondant sur les rapports étroits qui existent entre lignes de courant électrique et dislocations d'une part, induction magnétique et contraintes de l'autre [3]. Rappelons qu'une telle analogie a souvent été évoquée dans l'étude des dislocations dans les solides (cf. par exemple, de Wit [4]). Elle présente cependant dans ce cas une certaine difficulté, car elle demande d'identifier le vecteur de Burgers $b$, grandeur vectorielle, au courant $I$, grandeur scalaire. Mais dans le cas des smectiques le vecteur de Burgers est essentiellement un scalaire, puisqu'il ne présente de composante non nulle que selon la normale aux couches. Ainsi, dans le smectique fortement déformé où les couches prennent des formes proches de celles de cyclides de Dupin [5], la définition raisonnable de la grandeur caractéristique d'une ligne de dislocation est en termes de la phase $\varphi$ des couches; $\varphi$ est constant sur chaque couche, et tel que

$$
\text { d }|\operatorname{grad} \varphi|=d_{0}
$$

où $d$ est l'épaisseur de la couche déformée, $\dot{d_{0}}$ son épaisseur dans la situation d'équilibre planaire du smectique [6]. Définissons le nombre de Burgers par l'intégrale suivante prise sur un circuit $(\gamma)$ entourant la ligne :

$$
n=\frac{1}{d_{0}} \int_{\gamma} \mathrm{d} \varphi=\int_{\gamma} \frac{\mathbf{n}(\mathbf{r}) \cdot \mathrm{d} \mathbf{l}}{d}
$$

où $\mathbf{n}(\mathbf{r})$ est la normale unitaire à la couche, prise dans la même direction que $\nabla \varphi$ par convention, et dl l'élément d'arc selon $(\gamma)$. On notera que le nombre de Burgers dépend de l'orientation $\mathbf{n}(\mathbf{r})$ donnée aux couches, et change de signe lorsque $\mathbf{n}$ change de signe. Quant à l'orientation de $(\gamma)$, elle est liée, comme pour les dislocations dans les solides cristallins, à l'orientation (qui reste arbitraire) de la ligne de dislocation. Il y a donc deux arbitraires sur le signe de $\mathbf{n}$, alors qu'il n'y en a qu'un sur le signe de $I$, mais il est clair que l'analogie est ici plus profonde que dans le cas des dislocations dans les solides.

Le nombre de Burgers est bien l'analogue du courant $I$, si l'on convient d'identifier avec Pershan [3] 
le vecteur $\mathbf{m}(\mathbf{r})=\frac{\mathbf{n}(\mathbf{r})}{d}$ au champ magnétique $\mathbf{H}$ créé par la ligne, et le vecteur rot $\mathbf{m}$ à la densité de courant $\frac{4 \pi}{c} \mathbf{J}(\mathbf{r})$, qui obéit à l'équation de Maxwell

$$
\operatorname{rot} \mathbf{H}=\frac{4 \pi}{c} \mathbf{J}
$$

rot $\mathbf{m}$ est donc essentiellement une densité de dislocations. On a en effet

$$
\int_{\Sigma} \operatorname{rot} \mathbf{m} \cdot \mathrm{d} \boldsymbol{\Sigma}=\int_{\gamma} \mathbf{m} \cdot \mathrm{d} \mathbf{l}=n
$$

où $\Sigma$ est une surface quelconque limitée par $\gamma$, la composante $\alpha_{i}=\varepsilon_{i j k} m_{k, j}$ de rot $\mathbf{m}$ est donc le nombre de défauts qui percent perpendiculairement un élément de surface perpendiculaire à la direction $\mathrm{O} X_{i}$. En pratique, il sera plus intéressant de connaître les composantes de rot $\mathbf{m}$ parallèles à $\mathbf{n}$ (dislocations vis) et perpendiculaires à $\mathbf{n}$ (dislocations coin),

$$
\begin{array}{ll}
\mathbf{n} \operatorname{rot} \mathbf{m}=\alpha_{\mathbf{v}} & \text { densité de dislocations vis } \\
\mathbf{P} \operatorname{rot} \mathbf{m}=\alpha_{\mathbf{c}} & \begin{array}{l}
\text { densité }(2 \text { composantes }) \text { de disloca- } \\
\text { tions coin. }
\end{array}
\end{array}
$$

où $P_{i j}=\delta_{i j}-n_{i} n_{j}$ est l'opérateur de projection sur la couche.

Généralement, en raison de l'éq. (1), rot $\mathbf{m}$ est nul presque partout, sauf le long de lignes de singularités. On sera donc amené à poser

$$
\boldsymbol{\alpha}=\operatorname{rot} \mathbf{m}=n \mathbf{t} \delta\left(\mathbf{r}-\mathbf{r}_{0}\right)
$$

où $n$ est le nombre de Burgers de la ligne de tangente unitaire $\mathbf{t}$ passant par $\mathbf{r}_{0}$.

Pershan, se limitant au voisinage de la structure en couches planaires, identifie les trois composantes non nulles du tenseur des contraintes

$$
\begin{aligned}
& \sigma_{13}=-K_{1} \frac{\partial}{\partial x}\left(\frac{\partial^{2} u}{\partial x^{2}}+\frac{\partial^{2} u}{\partial y^{2}}\right) \\
& \sigma_{23}=-K_{1} \frac{\partial}{\partial y}\left(\frac{\partial^{2} u}{\partial x^{2}}+\frac{\partial^{2} u}{\partial y^{2}}\right) \\
& \sigma_{33}=B \frac{\partial u}{\partial z}
\end{aligned}
$$

à l'induction $\frac{1}{4 \pi} \mathbf{B}$, l'équation d'équilibre

$$
\sigma_{i_{3}, i}=0
$$

étant bien l'analogue de l'équation de Maxwell

$$
\operatorname{div} \mathbf{B}=0
$$

et de plus $\mathbf{m}$ et $\sigma_{i_{3}, i}$, comme $\mathbf{B}$ et $\mathbf{H}$, étant des grandeurs thermodynamiquement conjuguées, puisqu'on peut écrire que dans une variation infiniment petite du système l'énergie libre varie d'une quantité $\delta F$ donnée par

$$
\delta F=\int \sigma_{i_{3}} \delta m_{i} \mathrm{~d} V
$$

équation analogue à

$$
\delta F=-\frac{1}{4 \pi} \int \mathbf{B} \mathrm{d} \mathbf{H} \mathrm{d} V .
$$

La force de configuration qui s'exerce sur une ligne est de même l'analogue de la force de Lorentz qu'exerce l'induction sur le courant électrique, et on a :

$$
F_{i}=\varepsilon_{i j k} \sigma_{j, 3} \alpha_{k}
$$

En fait, il n'y a pas de difficultés à étendre au cas général de l'élasticité covariante [6] cette intéressante analogie proposée par Pershan. Montrons qu'il convient alors d'identifier à $-\frac{1}{4 \pi} \mathbf{B}$ le vecteur $\mathbf{S}$ introduit par Kléman et Parodi [6], défini par l'équation :

$$
\mathbf{S}_{i}=\frac{\partial(\rho f)}{\partial \varphi_{, i}}+\frac{P_{i j}}{|\nabla \varphi|}\left\{\left(\frac{\partial(\rho f)}{\partial n_{j, k}}\right)_{, k}-\frac{\partial \rho f}{\partial n_{j}}\right\}
$$

et qui obéit à l'équation

$$
\operatorname{div} \mathbf{S}=0
$$

$\rho f$ étant la densité d'énergie libre, exprimée en fonction des dérivées de la phase $\varphi$ et des composantes et des gradients de $\mathbf{n}$. En fait, $\nabla \varphi$ et $\mathbf{n}$ étant liés (n $=\nabla \varphi /|\nabla \varphi|)$, on peut exprimer $\rho f$ uniquement en fonction de $\varphi$ et de ses dérivées. Il est alors aisé de montrer que dans une variation petite de $\nabla \varphi$, la variation d'énergie libre s'écrit :

$$
\delta F=\int S_{i} \delta \varphi_{i} \mathrm{~d} V+\int \frac{P_{i k}}{|\nabla \varphi|} \frac{\partial(\rho f)}{\partial n_{i, j}} \delta \varphi_{, k} v_{j} \mathrm{~d} \Sigma
$$

les $v_{j}$ étant les cosinus directeurs de la normale à la frontière du volume $V$.

On notera que dans la dérivation de (11), les variations virtuelles $\delta \varphi$ de la phase, seule variable du problème, entraînent des variations corrélatives $\delta u_{i}=-\delta \varphi n_{i}$ du fluide. $\varphi$ ne doit donc pas être interprétée ici comme une variable de perméation [6], dont les variations pourraient se faire à fluide invariable.

La formule (11) montre que $\mathbf{S}$ est thermodynamiquement conjugué de $\nabla \varphi$. On dérive donc immédiatement une généralisation de la formule de Peach et Koehler dans le cas non linéaire

$$
\mathbf{F}=\mathbf{S} \wedge \text { rot } \mathbf{m}
$$

soit, pour la force de configuration s'exerçant sur une dislocation isolée de nombre de Burgers $n$ et de tangente unitaire $\mathbf{t}$

$$
\mathbf{F}=n d_{0} \mathbf{S} \wedge \mathbf{t}
$$


où $\mathbf{S}$ est le champ de contraintes (vectoriel) existant à l'emplacement de la ligne. Mais ici on ne peut en général distinguer dans $\mathbf{S}$ les contributions venant de contraintes appliquées, de la ligne elle-même, ou des autres lignes. Pour nous éclairer sur cette difficulté, qui vient de la non-linéarité, il est intéressant de retrouver l'éq. (13) directement.

En utilisant des intégrations par parties, et en tenant compte de l'éq. (10) on peut écrire $\delta F$ sous la forme d'une intégrale de surface uniquement :

$$
\delta F=\int\left(S_{j} \delta \varphi+K_{1} \operatorname{div} \mathbf{n} \delta n_{j}\right) v_{j} \mathrm{~d} \Sigma
$$

Dans cette équation on a donné à l'intégrale de l'éq. (11) la forme précise qu'elle a lorsqu'on prend :

$$
\rho f=\frac{1}{2} B(1-|\nabla \varphi|)^{2}+\frac{1}{2} K_{1}(\operatorname{div} \mathbf{n})^{2} .
$$

Supposons une ligne de dislocation $\mathrm{L}$, de surface de coupure $\Sigma_{L}$, et modifions la forme de la ligne en déplaçant $\Sigma_{\mathrm{L}}$ en $\Sigma_{\mathrm{L}}+\mathrm{d} \Sigma_{\mathrm{L}}$. Ceci demande deux opérations :

- Une translation relative des couches au voisinage de la surface de coupure $\Delta \varphi=-n d_{0}$. Cette translation peut se faire sans faire tourner $\mathbf{n}$. Le travail nécessaire à la création de $\mathrm{d} \Sigma_{\mathrm{L}}$ peut s'exprimer comme une intégrale sur les lèvres de $\mathrm{d} \Sigma_{\mathrm{L}}$, à l'aide de la formule (14) où on fait $\delta n_{j}=0$. On a alors :

$$
\delta F=-\Delta \varphi(\mathbf{S} \wedge \mathbf{t}) \cdot \mathrm{du} \mathrm{d} l
$$

où $\mathrm{d} \Sigma_{\mathrm{L}}=\mathrm{dl} \wedge \mathrm{du}$ est assimilé à un rectangle infiniment petit de dimensions $\mathrm{d} l$ (selon $\mathbf{t}$, vecteur unitaire tangent à $\mathrm{L}$ ) et $\mathrm{d} u$ (selon une direction perpendiculaire à t). En identifiant $\delta F$ à l'opposé du travail de la force de configuration, soit

$$
\delta F=-\mathbf{F} . \mathrm{du} \mathrm{d} l,
$$

on retrouve bien l'éq. (13).

- Une rotation relative des couches $\Delta \omega=n^{\prime} \pi k$ si la ligne $\mathrm{L}$ contient un terme de dislocation de rotation. Nous n'avons pas tenu compte d'une telle possibilité jusqu'à présent. $\mathrm{Si}$ une telle dislocation existe, il faut décomposer son effet sur $\mathrm{d} \Sigma_{\mathrm{L}}$ en deux parties :

— un déplacement $\Delta \omega \wedge \cdot d u$,

- une rotation locale $\Delta \omega$ des molécules.

Si $\Delta \omega \wedge$ du est selon le plan des couches, ce qui implique un choix particulier de la surface de coupure $\Sigma_{\mathrm{L}}$, la variation de phase correspondante $\Delta \varphi=-(\Delta \omega \wedge$ du $) . n$ est nulle, et il n'y a pas de travail de la force de configuration correspondante. Considérons donc uniquement la rotation locale. Posons $\delta \mathbf{n}=\delta \boldsymbol{\omega} \wedge \mathbf{n}$. Il vient

$$
\delta F=K_{1} \operatorname{div} \mathbf{n} \varepsilon_{j k l} n_{l} \delta \omega_{k} \varepsilon_{j p q} t_{p} \mathrm{~d} u_{q} \mathrm{~d} l
$$

ce qui correspond, en identifiant $\delta \omega$ à $\Delta \omega$, à une force de configuration

$$
F=K_{1} \operatorname{div} \mathbf{n}(\mathbf{n} \wedge \Delta \omega) \wedge \mathbf{t} .
$$

Cette quantité n'a pas d'analogue électromagnétique. Elle n'est de plus pas d'un très grand intérêt dans le cas des smectiques, car sa dérivation suppose explicitement $\Delta \omega$ petit, ce qui n'est pas le cas. Elle nous remet cependant en mémoire ce fait que l'état de distorsion autour d'une dislocation de rotation dépend de sa surface de coupure $\left({ }^{1}\right)$.

En revanche, la dérivation de (13) est correcte, car $n d_{0}$ peut être petit à l'échelle de l'élasticité continue utilisée ici. On obtient quelques résultats supplémentaires en traitant la dislocation de translation comme un objet linéaire (du type petite perturbation) superposé à un fond non linéaire (un domaine confocal où les couches sont fortement courbées). Considérons alors la création, par une suite de processus infinitésimaux réversibles [7], d'une ligne $L$ dans un milieu fortement distordu de champ de contraíntes $\mathbf{S}_{0}$, et supposons que l'introduction de $\mathrm{L}$ produit un terme perturbatif $\delta \mathbf{S}_{\mathrm{L}}$. Il est alors clair que l'énergie de $\mathrm{L}$ s'écrit

$$
W_{\mathrm{L}}=n d_{0} \int_{\Sigma} \mathbf{S}_{0} \cdot v \mathrm{~d} \Sigma+\frac{1}{2} n d_{0} \int_{\Sigma} \delta \mathrm{S}_{\mathbf{L}} \cdot v \mathrm{~d} \Sigma
$$

et comprend deux termes qui ne dépendent que de $\mathrm{L}$, puisque $\mathbf{S}_{0}$ et $\delta \mathbf{S}_{\mathrm{L}}$ sont des vecteurs solénoïdaux. Le second est l'analogue du terme usuel obtenu en théorie linéaire [7]. Nous nommerons le premier - énergie d'installation $W_{\text {IL }}$ [8]. Il peut être négatif ou positif selon le signe de $n$, mais ne dépend pas des arbitraires de signe sur $n$, en raison de la dépendance linéaire de $\mathbf{S}_{0}$ en $\mathbf{n}$.

Plus précisément, supposons que la région considérée soit un domaine confocal. Si nous négligeons la dilatation des couches [6], on a :

$$
S_{0}=-K_{1} \mathbf{P} \cdot \nabla \operatorname{div} \mathbf{n}
$$

S est un vecteur qui est dans le plan des couches et a pour composantes, dans le repère local des lignes de courbure

$$
+K_{1} \frac{\sigma_{2, \alpha}}{A} ; \quad+K_{1} \frac{\sigma_{1, \beta}}{B}
$$

où $A, B, \mathrm{~d} \alpha, \mathrm{d} \beta$ sont les éléments de la forme différentielle fondamentale de la couche

$$
\mathrm{d} s^{2}=A^{2} \mathrm{~d} \alpha^{2}+B^{2} \mathrm{~d} \beta^{2}
$$

$\left({ }^{1}\right)$ Je remercie M. le Pr. Friedel pour de stimulantes discussions à ce sujet. 
et $\sigma_{1}=1 / R_{1}, \sigma_{2}=1 / R_{2}$ les courbures principales. On a utilisé les relations

$$
\begin{aligned}
\operatorname{div} \mathbf{n} & =-\left(\sigma_{1}+\sigma_{2}\right), & & \text { valable sur toute surface } \\
\sigma_{2, \alpha} & =\sigma_{2, \beta}=0 & & \text { sur une cyclide de Dupin }
\end{aligned}
$$

l'énergie d'installation d'une dislocation coin est donc nulle, à l'approximation faite $\left(\varepsilon_{0}=0\right)$. De toute manière, on sait que les dislocations coin ont des vecteurs de Burgers considérables dans les smectiques $[9,10]$ (ce qui fait d'ailleurs qu'elles sont par nature très semblables aux domaines confocaux). La théorie quasi linéaire que nous développons ne leur est donc pas applicable.

L'expression (18) s'applique aux dislocations vis de petits vecteurs de Burgers. On peut donc concevoir que, pour certains systèmes de couches solutions de $\operatorname{div} \mathbf{S}_{0}=0, W_{\mathrm{L}}$ devient négatif, bien que le second terme $\left(\sim n^{2} d_{0}^{2}\right)$ soit essentiellement positif. Nous n'avons pas étudié ces solutions en détail. Mais on peut s'attendre à ce que certaines géométries à courbures particulières $\sigma_{1}+\sigma_{2}$ soient déstabilisées par la présence de dislocations vis et que d'autre part, dans une région à courbure favorable à leur présence, les dislocations soient essentiellement d'un même signe. D'autre part il est vraisemblable que les situations à $\sigma_{1}+\sigma_{2}=0$ (surfaces minima) soient de stabilité indifférente à la présence de dislocations vis, $W_{\mathrm{IL}}$ étant nul dans ce cas. Ceci permettrait d'expliquer pourquoi les courbures observées expérimentalement sont, sauf cas exceptionnel, négatives $\left(\sigma_{1}, \sigma_{2}<0\right)$. Nous montrerons dans un autre article que la relation ici suggérée entre dislocations vis et surfaces minima est en fait fondamentale.

Notons finalement que l'énergie d'interaction entre deux lignes peut s'écrire, dans la même approximation quasi linéaire :

$$
W_{\mathrm{I}}=n d_{0} \int_{\Sigma} \delta \mathbf{S}_{\mathrm{L}^{\prime}} \cdot \mathbf{v} \mathrm{d} \Sigma=n^{\prime} d_{0} \int_{\Sigma} \delta \mathbf{S}_{\mathrm{L}} \cdot v^{\prime} \mathrm{d} \Sigma^{\prime}
$$

Notre dernière remarque portera sur la non-linéarité : il est en effet intéressant de reconnaître qu'une formule comme celle de Peach et Köhler, habituellement considérée comme propre à une théorie linéaire, appartient fondamentalement, de par l'analogie avec les lignes de courant, à une théorie non linéaire. Son existence est seulement liée à l'existence d'une énergie libre et de variables d'état. Mais comme nous l'avons vu, son interprétation est alors moins immédiate que dans le cas linéaire.

Plus précisément, notons que la raison essentielle pour laquelle il existe une force de Lorentz est que B et $\mathbf{H}$ sont des variables thermodynamiquement conjuguées et il en est de même pour rot $\mathbf{m}$ et $\mathbf{S}$. Mais la relation précise (la loi de comportement) qui existe entre $\mathbf{B}$ et $\mathbf{H}$, ou entre $\varphi$ et $\mathbf{S}$, ne joue aucun rôle dans la dérivation. C'est la raison pour laquelle nous avons pu obtenir les résultats qui précèdent, malgré la non-linéarité.

\section{Bibliographie}

[1] De GenNes, P. G., Physics of liquid Crystals (Oxford University Press) 1974.

[2] KlÉman, M., J. Physique 35 (1974) 595.

[3] Pershan, P. S., J. Appl. Phys. 45 (1974) 1590.

[4] De Wit, R., Solid State Phys. 10 (1960) 249.

[5] Friedel, G., Ann. Phys. 18 (1922) 273.

[6] Kléman, M. et Parodi, O., J. Physique 36 (1975) 671.

[7] Friedel, J., Dislocations (Pergamon Press) 1964, chapitre II.

[8] Nous préférons le terme Energie d'installation, à celui d'énergie d'interaction, car rien ne nous permet d'affirmer qu'il y a égalité des énergies de ce type selon que l'on introduise la ligne $\mathbf{L}$ sur du smectique déformé, en domaines focaux, ou que l'on déforme le smectique après avoir d'abord créé L. Nous remercions J. P. Poirier pour une intéressante discussion à ce sujet.

[9] Williams, C. et Kléman, M., J. Physique Colloq. 36 (1975) C1-389.

[10] Kléman, M., Colliex, C. et Veyssié, M., Adv. chem. Ser. (1975) à paraître. 